\title{
Modeling of T/C fatigue test with boundary element method and linear fracture mechanics
}

\author{
A. Dansou ${ }^{1}$, S. Mouhoubi ${ }^{1}$, C. Chazallon ${ }^{1}$ and M. Bonnet ${ }^{2}$ \\ ${ }^{1}$ ICUBE, UMR 7357, INSA de Strasbourg, 24 boulevard de la Victoire, 67084 Strasbourg \\ 2 POEMS, UMR 7231, ENSTA Paris, 828 boulevard des Maréchaux, 91120 Palaiseau \\ anicet.dansoudinsa-strasbourg. fr
}

\begin{abstract}
This work presents the simulation of 3D crack propagation in samples in order to determine fatigue life. The modellings have been achieved by using MBEMv3.0: a fast software based on the Symmetric Galerkin Boundary Element Method (SGBEM) accelerated with the Fast Multipole Method (FMM) in 3D elasticity. Fatigue crack propagation has been simulated with Paris law. We present the simulations of a tensile/compression fatigue test on cylindrical samples of a semi-coarse asphalt concrete considered as homogeneous, containing very small cracks. When the number of cycles increases, the cracks propagate, and we can observe a loss of rigidity of the sample. Parametric studies of the modelling parameters have been performed where the damage evolutions exhibit a typical shape that proves that, for asphalt concrete materials subjected to $\mathrm{T} / \mathrm{C}$ fatigue test, the shape of the fatigue test curve is mainly governed by biasing effects at the beginning then by mechanical damage at the end.
\end{abstract}

Keywords: Fatigue life, Crack propagation, SGBEM, FMM

\section{Introduction}

Fatigue cracks are among the major causes of structural degradation. Experimental methods have been widely used since a long time to predict their propagation. However numerical methods are very interesting because some destructive experimental tests, which can entail important costs and long delays, can be avoided. This work presents the simulation of 3D crack propagation in order to determine fatigue life by using a fast-numerical software developed in our laboratory: MBEMv3.0. This software is based on two sophisticated methods of integral equations: The Boundary Element Method (BEM) and the Fast Multipole Method (FMM).

Although the Finite Element Method (FEM) is the best-known method in fracture mechanics, BEM has significant advantages, for example, the reduction by one of thr problem dimension. For a $3 \mathrm{D}$ problem, only the boundary and crack surfaces are discretized, rather than the volume. Moreover, meshes can easily be generated and design changes do not require a complete remeshing; this is very suitable for crack problems. One of the main drawbacks of BEM is that the solution matrix resulting from the formulation is unsymmetric and fully populated, whereas, the FEM matrices 
are usually much larger but sparse. An interesting approach of BEM is the Symmetric Galerkin BEM (SGBEM) which is based on a variational (weak) version of the integral equations. It entails double surface integrations but leads to matrix operators which exhibit symmetry and sign-definiteness. SGBEM is used in many works, see for example Gray and Paulino [1], Frangi [2], or Pham et al. [3].

Over recent decades, the performance of boundary analysis is further improved with the Fast Multipole Method (FMM) introduced by Rokhlin [4]. The classical bottlenecks of the BEMs, arising from the fully populated matrix, are alleviated as the FMM splits all element integrals into near-field and far-field interactions, the latter being clustered in a recursive, multilevel fashion. This process results in a lessened storage complexity, typically defined by the sparse near-field matrix, and faster solution based on iterative solvers. This makes boundary element analysis applicable to large BEM models with very good performance, see for example Yoshida [5], Chaillat [6], Pham et al. [3] or Trinh et al. [7].

\section{Crack propagation simulation by MBEMv3.0}

Based on SGBEM and FMM, Pham [8] and then Trinh [9] developed a Fortran code for 3D elasticity problems, called MBEMv2.0. This software inherits a number of innovative algorithms from the boundary element community, such as the singular integration schemes, the index of severity for adjusting the Gaussian quadrature density to the interelement distance, the nested Flexible GMRES (FGMRES) which uses the near-interaction matrix as a preconditioner and the extension to multizone configurations of the SGBEM. These features and attendant performance enhancements are reported in [7, 9]. Matrix-vector operations are performed using subroutines from the BLAS library. The FGMRES algorithm used is available at www.cerfacs.fr and described.

A new version of the code noted MBEMv3.0, is developed by Dansou et al. in [10, $11,12]$. In this version, multiple strategies have been proposed and implemented for performance enhancement, including a data reusing technique, a shared memory parallelism using OpenMP directives and the proposal of a new sparse matrix method. The enhanced code has been run on various large-scale tests $\left(\mathrm{N}=\mathrm{O}\left(10^{6}\right)\right)$ and has proved to be very robust and of excellent accuracy. The time speedup compared to MBEMv2.0 has exceeded 50 in some crack propagation simulations. For example, ten propagation cycles of 64 circular cracks in a cube sample are simulated in two hours on a 20-core computer. The new version can deal with surface breaking cracks, complex multizone problems and proposed a direct coupling with FEM to simulate thin structures.

In MBEM v3.0, the Paris law is used to simulate crack propagation. Abundant experimental evidence supports the view that the crack growth rate can be correlated with the cyclic variation in the stress intensity factors (SIFs), e.g. through: 


$$
\frac{d a}{d N}(s)=A \Delta K^{m}(s)
$$

where $s$ is the arc length coordinate along the crack front (Fig 1), $N$ is the number of loading cycles, $d a / d N$ is the fatigue crack advancement rate per cycle, $\Delta K=K^{\max }-K^{\min }$ is the SIF range for the current cycle, while $A$ and $m$ are parameters that depend on the material, environment, frequency, temperature and stress ratio. The SIFs are evaluated through extrapolation from the computed displacement discontinuity field. For example, $K_{I}$ is evaluated by:

$$
K_{I}^{2}=\frac{\mu}{8(1-v)} \sqrt{\frac{2 \pi}{a}}\left(4 \phi^{5}-\phi^{1}\right) \cdot n
$$

where $K_{I}^{2}$ is the mode I SIF at the node $2, \phi^{1}, \phi^{5}$ are the nodal crack opening displacements at nodes 1 and 5 , the nodes being numbered as shown in Fig 1. The geometrical advance of the crack (Fig.1) is described by moving points of the crack front in the local plane orthogonal to the front. The direction $\theta_{0}$ of the crack advancement is assumed to be given by the maximum circumferential stress criterion:

$$
\tan \frac{\theta_{0}}{2}=\frac{1}{4}\left[\frac{K_{\text {Ieff }}}{K_{I I}}-\operatorname{sign}\left(K_{I I}\right) \sqrt{\left(\frac{K_{\text {Ieff }}}{K_{I I}}\right)^{2}+8}\right]
$$

where $K_{\text {Ieff }}=K_{I}+B\left|K_{I I I}\right|$ is an equivalent local mode I stress intensity factor which accounts for the tearing mode being active, $B$ being a material parameter. The length $\Delta a$ of the crack advancement is determined from the Paris law:

$$
\Delta a(s)=A\left(\Delta K_{I}^{2}(s)+K_{I I}^{2}(s)\right)^{m / 2} \Delta N
$$

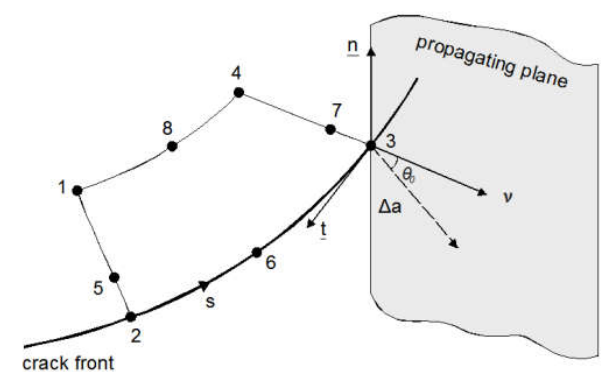

Fig 1: Crack front element in MBEMv3.0

\section{$3 \quad$ Fatigue test on cylindrical samples}

\subsection{Model}

We consider cylindrical samples of a semi-coarse asphalt concrete considered as homogeneous $(E=17000 \mathrm{MPa}, v=0.3)$. The sample of dimensions $\mathrm{R}=75 \mathrm{~mm}, \mathrm{H}=$ $150 \mathrm{~mm}$ contains 64 randomly oriented cracks with initial radius of $r_{c}=1 \mathrm{~mm}$, see Fig. 
2. The initial mesh features 3264 elements and 31494 DOFs. The material properties for the Paris law are $\mathrm{A}=10^{-8} \mathrm{~mm} /$ cycle and $\mathrm{m}=4.5$, see [13]. The tolerance for the iterative solver is set to $10^{-3}$. The tensile load is $\sigma=1.7 \mathrm{MPa}$ which corresponds to an initial strain $\varepsilon=10^{-4}$.

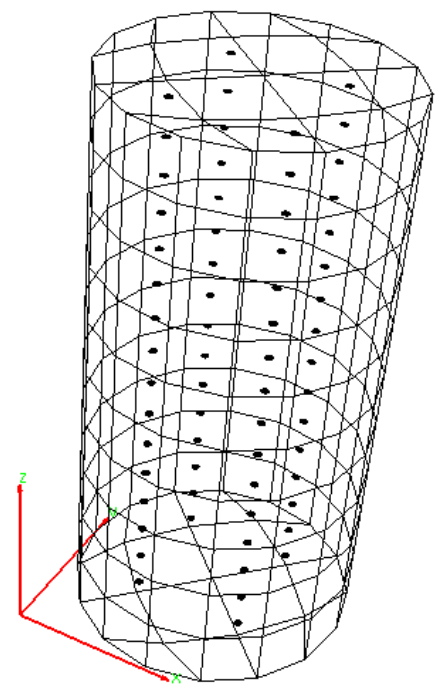

(a) Model with $r_{c}=1 \mathrm{~mm}$

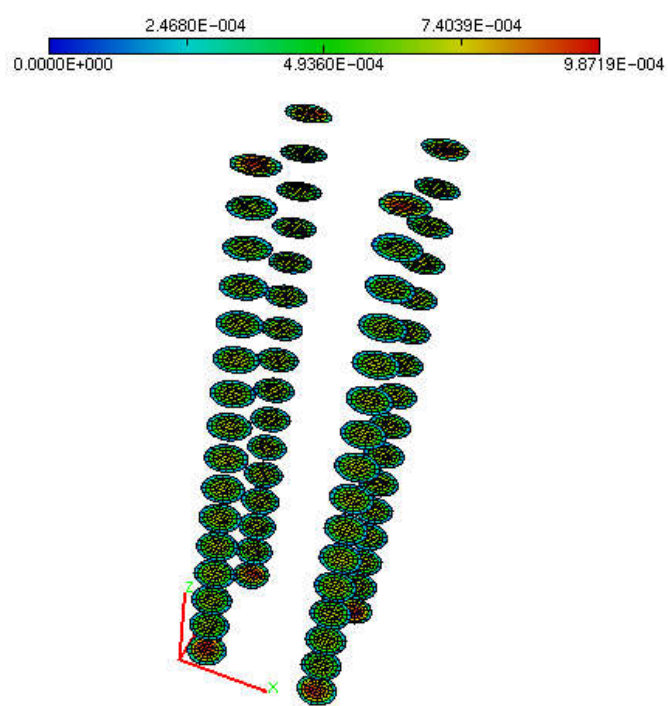

(b) Cracks in propagation

Fig 2: Model of a multi-cracked sample

\subsection{Results}

When the number of cycles increases, the cracks propagate, and we can observe a loss of rigidity of the sample. The decrease of the residual stiffness according to the number of loading cycles can be divided in two main stages.

The first stage can correspond to the initiation of the crack propagation. In this study, very small (radius $=1 \mathrm{~mm}$ ) initial cracks are generated. The residual rigidity at the initial state is equal to $99.5 \%$. This version of our code does not yet include criteria to initiate the propagation. As a result, the propagation speed is very low at the beginning of the simulation, the fatigue of the material is governed by biasing effects in this part. Table 1 gives a summary of the residual stiffness, the number of cycles and the crack size during this first part. A propagation from a radius of 1 to $2 \mathrm{~mm}$ requires 5 million of loading cycles and the residual stiffness decreases from $99.54 \%$ to $99.33 \%$. This very low speed is observed until a radius of $4 \mathrm{~mm}$ corresponding to a residual stiffness of $98.66 \%$. This limit is very interesting since it can permit to determine the threshold value of stress intensity factor below which fatigue cracks do not propagate. This threshold can then be compared to values obtained with various crack propagation criteria or with experiments. 
Table 1. Initiation of the crack propagation.

\begin{tabular}{ccc}
\hline Loading cycles & Crack radius & Residual stiffness (E/E0) \\
\hline 0 & No crack & $100 \%$ \\
- & $1 \mathrm{~mm}$ & $99,54 \%$ \\
5185400 & $2 \mathrm{~mm}$ & $99,33 \%$ \\
6419225 & $3 \mathrm{~mm}$ & $98,75 \%$ \\
6910735 & $4 \mathrm{~mm}$ & $98,66 \%$ \\
\hline
\end{tabular}

For the second stage, we monitor in Fig. 3the decrease of the residual stiffness. The final state of the first stage (cracked sample containing cracks of radius $4 \mathrm{~mm}$ ) is taken as the initial state for this second stage (point A in Fig 3). In Fig. 3, we clearly observe an acceleration of the rigidity loss, and can determine the fatigue life of the sample (number of cycles required to lose half of the initial rigidity). In this simulation, the fatigue life is $6.610^{5}$ cycles after stage 1 (initiation of the propagation) and the cracks have radii of $12-13 \mathrm{~mm}$ at this state (point B in Fig 3).

At the end of this stage, we observe a very fast decrease of the residual stiffness. This can be assimilated to the third region observed in experiments when the stress intensity factor approaches the fracture toughness and the material fails. This limit can help to determine a numerical value of the fracture toughness which can be compared to values obtained with experiments.

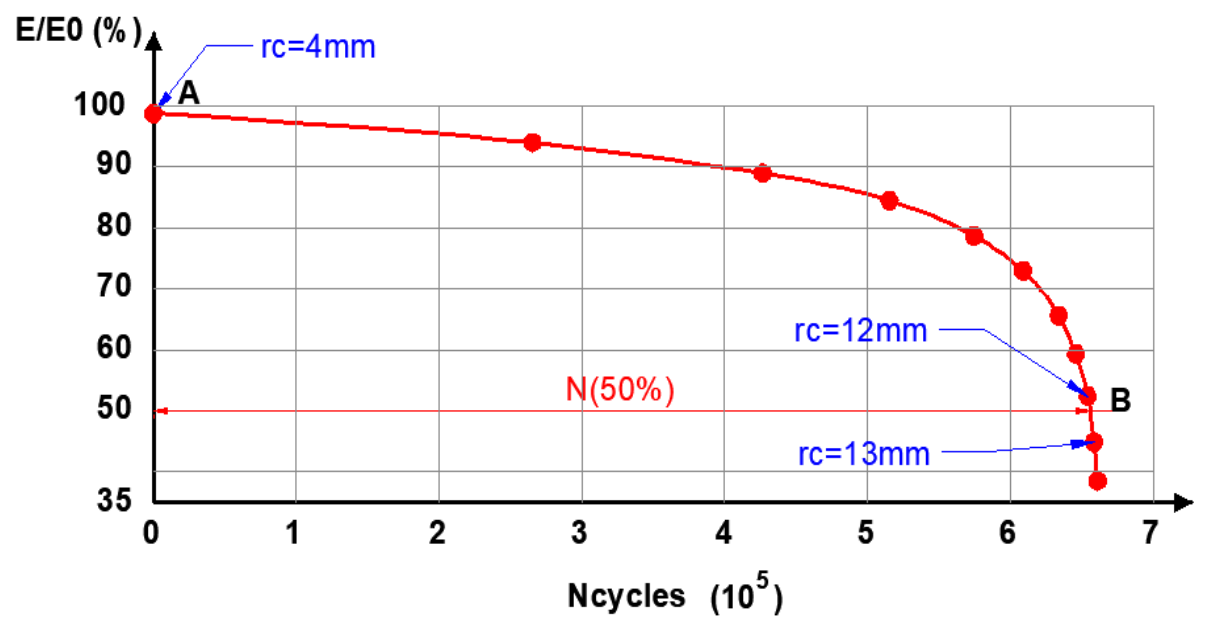

Fig 3: Evolution of residual stiffness of a multi-cracked sample 


\section{Conclusion}

This paper has shown MBEMv3.0 can help to simulate T/C fatigue tests. A Multicracked cylindrical sample of asphalt concrete is simulated for the purpose. The fatigue test curve is obtained by using Paris law. The shape of the curve is governed by biasing effects in a first stage, , and subsequently by mechanical damage.

\section{Acknowledgements}

This work is supported in part by the French National Research Agency (SolDuGri project ANR-14-CE22-0019) and in part by the region "Grand-Est, France".

\section{References}

1. Gray, L. J., Paulino, G. H.: Symmetric Galerkin boundary integral formulation for interface and multizone problems. Int. J. Num. Meth. Eng. 40, 3085-3101 (1997).

2. Frangi, A.: Fracture propagation in $3 \mathrm{~d}$ by the symmetric Galerkin boundary element method. Int. J. Fract. 116, 313-330 (2002).

3. Pham, D., Mouhoubi, S., Bonnet, M., Chazallon, C.: Fast multipole method applied to Symmetric Galerkin boundary element method for 3D elasticity and fracture problems. Engineering Analysis with Boundary Elements 36, 1838-1847 (2012).

4. Rokhlin, V.: Rapid solution of integral equations of classical potential theory. Journal of Computational Physics 60(2), 187-207 (1985).

5. Yoshida, K.: Applications of Fast Multipole Method to Boundary Integral Equation Method. PhD Thesis. Kyoto Univ., Japan, (2001).

6. Chaillat, S. : Méthode multipôle rapide pour les équations intégrales de frontière en élastodynamique 3-D. Application à la propagation d'ondes sismiques, $\mathrm{PhD}$ Thesis. Ecole des Ponts ParisTech, Champs-sur-Marne, France (2008).

7. Trinh, Q. T., Mouhoubi, S., Chazallon, C., and Bonnet, M.: Solving multizone and multicrack elastostatic problems: A fast multipole symmetric Galerkin boundary element method approach. Engineering Analysis with Boundary Elements 50, 486-495 (2015).

8. Pham, D. : Méthode multipôle rapide pour les équations intégrales variationnelles en élasticité tridimensionnelle et en mécanique de la rupture. Thèse. INSA de Strasbourg (2010).

9. Trinh, Q. T.: Modelling multizone and multicrack in three-dimensional elastostatic media: a Fast multipole Galerkin Boundary Element Method. Thèse. INSA de Strasbourg (2014).

10. Dansou, A., Mouhoubi, S., Chazallon, C., and Bonnet, M.: Modeling multi-crack propagation by the Fast Multipole Symmetric Galerkin BEM. Engineering Analysis with Boundary Eléments 106, 309-319 (2019).

11. Dansou, A., Mouhoubi, S., and Chazallon, C.: Optimizations of a fast multipole symmetric Galerkin boundary element method code. Numerical Algorithms (2019).

12. Dansou, A.: Fast Multipole Boundary Element Method approach for Multicracked Structures: Application to Road Pavement Reinforcement. Thèse. INSA de Strasbourg (2019).

13. Corté, J. F., Di Benedetto, H.: Matériaux routiers bitumineux: description et propriétés des constituants. Lavoisier, 2004. 